\title{
Health screening behaviors among adults with hereditary hemorrhagic telangiectasia in North America
}

\author{
Melanie Baxter, ScM, CGC ${ }^{1,6}$, Lori Erby, PhD, CGC1, Debra Roter, DrPH², \\ Barbara A. Bernhardt, MS, LCGC ${ }^{3}$, Peter Terry, MD ${ }^{4}$ and Alan Guttmacher, MD 5
}

\begin{abstract}
Purpose: This study aimed to identify factors that influence screening behaviors of adults with hereditary hemorrhagic telangiectasia (HHT).

Methods: Participants with a self-reported diagnosis of HHT were recruited from the HHT Foundation International, Inc.; the "HHT Awareness" Facebook group; and six HHT clinics. A cross-sectional mixed methods survey was administered to investigate the relationships among the Health Belief model constructs, the domains of illness representations, and HHT-specific screening behaviors consistent with recommended guidelines.
\end{abstract}

Results: A total of 320 participants reported rates of cerebral arteriovenous malformation (AVM) screenings, pulmonary AVM screenings, and HHT annual checkups that were 82.0, 67.1, and 56.5\%, respectively. Logistical regression analysis showed that perceived barriers $(\beta=-0.114, P<0.001)$, perceived susceptibility $(\beta=0.117$, $P<0.05)$, treatment control $(\beta=0.078, P<0.05)$, and emotional representations $(\beta=0.067, P<0.05)$ were significant predictors of HHT screening. Open-ended responses revealed perceived barriers to screening, including a lack of health-care providers (HCPs) familiar with and/or knowledgeable about HHT.

Conclusion: Our results reveal suboptimal screening rates among adults with HHT and identify several factors influencing these behaviors. We suggest that there is a need for increased provider education regarding $\mathrm{HHT}$ as well as approaches that providers can use to improve screening adherence.

Genet Med advance online publication 13 October 2016

Key Words: hereditary hemorrhagic telangiectasia; Health Belief Model; illness representation; perceived barriers; screening behaviors

\section{Background}

\section{INTRODUCTION}

Hereditary hemorrhagic telangiectasia (HHT) is an autosomaldominant multisystem vascular dysplasia characterized by arteriovenous malformations (AVMs). ${ }^{1}$ The most common clinical manifestations are spontaneous and recurrent nosebleeds and telangiectases (small AVMs) of the hands, face, mouth, and gastrointestinal (GI) mucosa. However, large AVMs can occur in the lungs (PAVMs) and brain (CAVMs), leading to stroke, brain abscess, and seizures, as well as in other organs, including the liver. Although nosebleeds are most often the initial symptom, AVMs can occur before the appearance of mucosal/ cutaneous telangiectases and leave seemingly unaffected family members at risk for life-threatening complications. HHT is a chronic condition; however, with early diagnosis followed by adequate screening and treatment, the major complications of this disorder can often be avoided and disability or even death can be prevented.

The international HHT medical management guidelines ${ }^{2}$ were published in 2011, and have not yet been updated. Screening protocols in the clinical setting may therefore differ as guidelines begin to lag behind the state of the science. The 2011 guidelines $^{2}$ recommend screening for CAVMs in children and adults once at the time of initial diagnosis and additional screening only if there are positive MRI findings. For PAVM screening, the experts recommend contrast transthoracic echocardiography (TTCE) in children and adults at the time of initial evaluation and additional screening every 5-10 years if the TTCE results are negative. The expert panel also recommends that patients consult with gastroenterologists to check for gastrointestinal bleeding and liver and vascular malformations and with otorhinolaryngologists (ENTs) for epistaxis assessment. Additionally, consulting a physician knowledgeable about HHT is key in the care of patients with HHT because inadequate knowledge can raise barriers to prevention, diagnosis, and treatment. Research to date suggests that nonadherence to screening or preventive treatment is an overarching problem in

${ }^{1}$ Social and Behavioral Research Branch, National Human Genome Research Institute, National Institutes of Health, Bethesda, Maryland, USA; ${ }^{2}$ Health, Behavior and Society, Johns Hopkins Bloomberg School of Public Health, Baltimore, Maryland, USA; ${ }^{3}$ Division of Translational Medicine and Human Genetics, Perelman School of Medicine, University of Pennsylvania, Philadelphia, Pennsylvania, USA; ${ }^{4}$ Division of Pulmonary and Critical Care Medicine, Johns Hopkins Hospital, Johns Hopkins University School of Medicine, Baltimore, Maryland, USA; ${ }^{5}$ The Permanent Fund for Vermont's Children, Burlington, Vermont, USA; ${ }^{6}$ Current address: GeneDx, Elmwood Park, New Jersey, USA. Correspondence: Melanie Baxter (melanie.baxter@gmail.com) 
many medical conditions, ${ }^{3-12}$ and chronic genetic diseases such as HHT are no exception. ${ }^{13,14}$

\section{Theoretical framework}

The Health Belief Model (HBM), which guided the framework of this study, has been widely used to explain individual differences in preventive health behavior. ${ }^{11,15-18}$ The model is based on the principle that people will take action to prevent, screen for, or control ill-health conditions if (i) they regard themselves as susceptible to the condition/threat, (ii) they believe the condition/threat would have potentially serious consequences, (iii) they believe that a course of action available to them would be beneficial in reducing either their susceptibility to or the severity of the condition/threat, and (iv) they believe that the anticipated barriers to taking the action are outweighed by the benefits. ${ }^{15}$

Although Verkerk et al. assessed screening adherence for PAVMs in patients with $\mathrm{HHT}^{19}$ and found suboptimal levelsonly $57.7 \%$ of patients underwent PAVM screening-they did not examine factors that influenced adherence. The goal of this study was to quantify and explore factors that influence screening behaviors in a population of adults with HHT in order to identify targets for clinical intervention.

\section{MATERIALS AND METHODS \\ Study population}

Participants were recruited from the HHT Foundation International, the Facebook HHT Awareness group, and six HHT clinics across the United States that agreed to assist with recruitment: the Yale School of Medicine, University of Utah Medical Center, Oregon Health \& Science University, Washington University School of Medicine, University of Pennsylvania, and Johns Hopkins Hospital. A snowball recruitment technique was used to recruit additional participants. Eligibility criteria were age $\geq 18$ years and self-reported diagnosis of HHT. Participants who completed the survey were given a $\$ 10$ gift card. The protocol was approved by the institutional review board of the National Human Genome Research Institute, and all participants provided informed consent.

\section{Study design}

The cross-sectional survey was based on a mixed-methods approach. The quantitative component consisted of assessment of illness representations, perceived susceptibility, perceived benefits and barriers, and self-efficacy (Rosenstock et al. ${ }^{20}$ proposed that self-efficacy-an individual's confidence in one's ability to take action - be added to the HBM as an independent variable based on evidence of its independent effect on behavior change). Illness representations were evaluated to take into account the individual's cognitive and emotional perceptions of their condition. We also assessed demographic information, including gender, age, marital status, income, education, race/ethnicity, insurance status, country of residence, parental status, and HHT-diagnostic status of children, as well as two measures constructed for this study: (i) response efficacy and (ii) assessment of HHT screening adherence. The qualitative component of the survey comprised six open-ended questions to further explore factors related to adherence.

\section{Measures}

IIIness representations. Illness representations were captured using the Illness Perception Questionnaire (IPQ-R), ${ }^{21}$ a theoretically derived measure that asks participants to consider their own personal views of how they perceive their current illness. The items are categorized by seven domains of illness representations-time line (acute/chronic, cyclical), consequences, personal and treatment control, coherence, emotional representations, identity, and cause-that have been validated by Moss-Morris et al. ${ }^{21}$ using eight illness groups. Psychometric analysis revealed that all of the IPQ-R subscales demonstrate good internal reliability. The Cronbach's alphas for each of the subscales ranged from 0.79 to 0.89 .

Health belief. The Champion Health Belief Model Scale (CHBMS) was developed with the intention of measuring the concepts of the HBM in numerous study populations and across various preventive health behaviors. The CHBMS has been shown to be reliable and valid, and there is evidence supporting its content and construct validity.2 The Cronbach's alpha measurements were 0.83 for perceived susceptibility, ${ }^{23}$ 0.85 for perceived barriers, ${ }^{23} 0.65$ for perceived benefits, ${ }^{23}$ and 0.87 for self-efficacy. ${ }^{24}$ The CHBMS was redesigned to be specific to HHT screening. The response efficacy scale was adapted and validated from previous research on breast cancer screening behavior ${ }^{25}$ and exercise behavior to reduce coronary heart disease ${ }^{26}$ In this study, a four-question measure assessing response efficacy focused on the benefits and barriers of screening the brain and lungs and seeing a health-care provider (HCP) knowledgeable about HHT.

HHT screening adherence measure. The HHT screening adherence measure consisted of three items that assessed past participation in screening related to HHT, including a cranial MRI ("When was the last MRI of your brain?"), PAVM screening ("When was the last time you had your lungs screened for your HHT?"), and annual follow-up with an HCP knowledgeable about HHT. The past behavior scores for each of the screening behaviors were summed and treated as a categorical variable (high, medium, and low). "High" adherers were participants who fulfilled all three recommended guidelines, "medium" adherers were participants who are late for PAVM screening or late for annual checkup but have undergone these in the past, and "low" adherers are participants who have completely neglected at least one aspect.

Open-ended questions. Six questions were posed to elicit information regarding additional possible barriers and benefits to HHT screening. The questions were "What do you consider to be the most important benefit(s) to having an MRI of your brain?", "What things could/do keep you from getting an MRI 
Table 1 Description of study sample

\begin{tabular}{|c|c|c|c|}
\hline Sociodemographic variable & & Total $\boldsymbol{N}$ & Percentage \\
\hline \multirow{2}{*}{ Gender } & Female & 215 & 72.9 \\
\hline & Male & 80 & 27.1 \\
\hline \multirow[t]{2}{*}{ Marital status } & Married & 226 & 77.4 \\
\hline & Not married & 66 & 22.6 \\
\hline \multirow[t]{4}{*}{ Highest level of education } & High school/GED & 57 & 19.5 \\
\hline & Some college & 66 & 22.6 \\
\hline & College degree & 102 & 34.9 \\
\hline & Postgraduate & 67 & 22.9 \\
\hline \multirow[t]{2}{*}{ Race } & Caucasian & 277 & 94.2 \\
\hline & Not Caucasian & 17 & 5.8 \\
\hline \multirow[t]{2}{*}{ Ethnicity } & Hispanic & 8 & 2.8 \\
\hline & Not Hispanic & 273 & 97.2 \\
\hline \multirow[t]{5}{*}{ Annual household income } & $<\$ 25,000$ & 25 & 9.3 \\
\hline & $\$ 25,000-49,999$ & 45 & 16.8 \\
\hline & $\$ 50,000-74,999$ & 69 & 25.7 \\
\hline & $\$ 75,000-99,999$ & 36 & 13.4 \\
\hline & $>\$ 100,000$ & 93 & 34.7 \\
\hline \multirow[t]{2}{*}{ Insurance status } & Insurance & 267 & 91.4 \\
\hline & No insurance & 25 & 8.6 \\
\hline \multirow[t]{2}{*}{ Country of residence } & United States & 231 & 78.6 \\
\hline & Outside United States & 63 & 21.4 \\
\hline \multirow[t]{2}{*}{ Parental status } & Yes & 227 & 76.7 \\
\hline & No & 69 & 23.3 \\
\hline \multirow[t]{2}{*}{ Child's HHT status } & Yes & 29 & 12.8 \\
\hline & No/unknown & 198 & 87.2 \\
\hline Demographic variable & & Mean (SD) & Range \\
\hline Age, years & & $49.5( \pm 13.7)$ & $18-82$ \\
\hline Years since HHT diagnosis & & $29.25( \pm 14.6)$ & $0-69$ \\
\hline Recruitment source ${ }^{a}$ & & Total N (\%) & \\
\hline HHT foundation direct mailing & & $100(31.7)$ & \\
\hline HHT foundation e-mail notification (e-blast) & & $82(26.0)$ & \\
\hline HHT Foundation website & & $77(24.4)$ & \\
\hline Family member & & $33(10.5)$ & \\
\hline Facebook "HHT Awareness" group & & $17(5.4)$ & \\
\hline HHT clinic & & $4(1.3)$ & \\
\hline Friend & & $2(0.6)$ & \\
\hline
\end{tabular}

${ }^{a} N=315$; five participants did not report the source of recruitment.

GED, general equivalency diploma; HHT, hereditary hemorrhagic telangiectasia.

of your brain?", "What do you consider to be the most important benefit(s) to having your lungs screened?," "What things could/ do keep you from getting your lungs screened?," "What do you consider to be the most important benefit(s) of seeing a healthcare provider who is knowledgeable about HHT?," and "What things could/do keep you from seeing a health-care provider who is knowledgeable about HHT?"

\section{Data analysis}

Data were analyzed using SPSS 16.0, and the primary outcome variable was the three-category measure of screening adherence. The polytomous universal model (PLUM) ${ }^{27}$ under a cumulative odds model was used to determine the association of illness representations and Health Belief Model constructs with the level of HHT screening adherence after controlling for potential confounding variables. Each potential confounder (all demographic variables, age at diagnosis, parental status, and HHT diagnostic status of children) was tested as a predictor of adherence using ANOVA or PLUM, as appropriate. Relationships that resulted in $P \leq 0.20$ were included in all subsequent multivariate regression models. Backward elimination was used to test for the association of one covariate of the outcome measure while controlling for other covariates. Our power analysis for a logistic regression (two-tailed test) using an alpha of 0.05 and $80 \%$ power suggested that a sample size 
Table 2 Scale descriptives

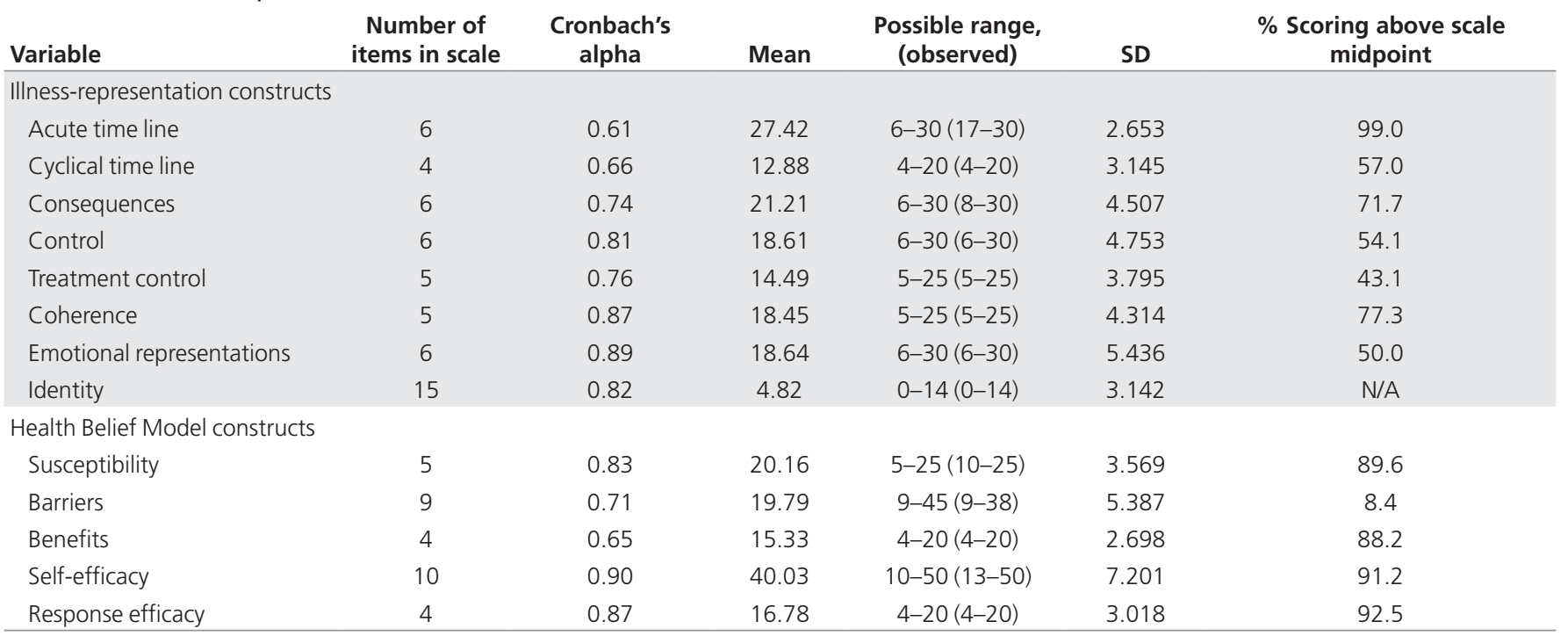

Table 3 Multiple ordinal logistic regressions: prediction of hereditary hemorrhagic telangiectasia screening adherence

\begin{tabular}{|c|c|c|c|}
\hline \multirow{2}{*}{\multicolumn{2}{|c|}{ Susceptibility }} & $\begin{array}{c}\text { Unadjusted } \\
\text { model } \beta \\
\text { Estimate }\end{array}$ & $\begin{array}{c}\text { Final model } \\
\beta \text { Estimate }\end{array}$ \\
\hline & & 0.074 & $0.117 *$ \\
\hline \multicolumn{2}{|l|}{ Barriers } & $-0.096 *$ & $-0.114 * *$ \\
\hline \multicolumn{2}{|l|}{ Self-efficacy } & 0.030 & - \\
\hline \multicolumn{2}{|l|}{ Response efficacy } & 0.025 & - \\
\hline \multicolumn{2}{|l|}{ Consequences } & 0.011 & - \\
\hline \multicolumn{2}{|l|}{$\begin{array}{l}\text { Emotional } \\
\text { representations }\end{array}$} & 0.050 & $0.067^{*}$ \\
\hline \multicolumn{2}{|l|}{ Identity } & 0.083 & - \\
\hline \multicolumn{2}{|l|}{ Treatment control } & 0.063 & $0.078^{*}$ \\
\hline \multirow[t]{4}{*}{ Education } & High school/GED & $-1.142 *$ & $-1.234^{*}$ \\
\hline & Some college & -0.605 & -0.619 \\
\hline & College degree & $-0.751 *$ & $-0.807 *$ \\
\hline & Postgraduate (ref.) & 0 & 0 \\
\hline \multirow[t]{2}{*}{ Insurance status } & No & -0.937 & -1.001 \\
\hline & Yes (ref.) & 0 & 0 \\
\hline \multicolumn{2}{|c|}{ Cox and Snell pseudo- $R^{2}$} & 0.227 & 0.212 \\
\hline \multicolumn{2}{|l|}{$\begin{array}{l}\text { Test of parallel } \\
\text { lines significance }\end{array}$} & 0.356 & 0.004 \\
\hline
\end{tabular}

Values shown in bold are significant.

GED, general equivalency diploma.

${ }^{*} P<0.05,{ }^{*} P<0.001$.

of 268 would be sufficient to detect a small effect (odds ratio $=1.5$ ).

Brief thematic analysis was used to explore data collected from the open-ended questions. Responses were analyzed for overarching themes and then grouped accordingly. Major themes were further analyzed for subcategories and frequencies of common themes were calculated. Responses representing more than one theme were coded for each theme characterized.

\section{Study participants}

\section{RESULTS}

Three-hundred twenty surveys were received: 311 electronic and 9 paper versions. Thirty surveys were submitted incomplete, but these were included in analyses for which survey data were available in order to maximize the amount of data for each analysis. The most cited source of recruitment for participants was the HHT Foundation International (82.1\%).

The average age was 49.5 years $( \pm 13.7)$ and ranged from 18 to 82 years. The participants varied widely in how long they had known their HHT diagnosis, from less than 1 year to 69 years, with a mean of 29.25 years $( \pm 14.6)$. The study population was mostly female (72.9\%), white (94.2\%), not of Hispanic origin (97.2\%), and married (77.4\%). The majority of participants $(57.8 \%)$ had a college or postgraduate degree. Additionally, the majority of participants were parents (76.7\%), of whom $12.8 \%$ reported that at least one of their children had been diagnosed with HHT. Table 1 summarizes the characteristics of the study sample.

\section{Illness representations and Health Belief Model variables}

The descriptive statistics and Cronbach's alpha values for all key predictor variables are shown in Table 2. The internal reliability of each of the IPQ-R scales was acceptable, with the exception of the acute time line and cyclical time line scales $(\alpha=0.61$ and 0.66 , respectively). The percentages of participants scoring above the midpoint for each scale of the IPQ-R and the HBM scale are also shown; this provides an indication of the proportion of participants holding particularly strong views about the construct being measured by each particular scale. For example, most participants reported a high degree of personal understanding about their condition, with $77.3 \%$ scoring above the midpoint on the IPQ coherence scale. Almost all participants scored above the midpoint on the IPQ acute time line scale, indicating that they consider their illness chronic, which 
is true for HHT. The majority of participants also perceived their HHT as having particularly severe consequences for their lives. Single-item analysis revealed that $84 \%$ agreed that "My HHT is a serious condition," and $67 \%$ of participants said that HHT has major consequences for their lives.

Exactly $50 \%$ of participants scored above the midpoint on the emotional representations scale (assessing negative emotions associated with HHT), with reported feelings including depression or being upset (35\%), anger (29\%), fear (41\%), and anxiety (48\%) regarding their condition.

\section{Reported adherence to HHT screening}

When asked about past cranial MRI behavior, $82 \%$ reported having undergone a cranial MRI at some point in their lifetime, $17.3 \%$ reported never having undergone a cranial MRI, and $0.7 \%$ were unsure. When asked about past lung screening behavior, $67.1 \%$ reported having undergone screening for pulmonary PAVMs within the past 5 years, $18.1 \%$ had undergone screening 5 or more years previously, and $14.9 \%$ had never had their lungs screened or were unsure. The final component of the screening-adherence measure asked about past follow-up with an HCP familiar with HHT. The majority of study participants (56.5\%) had seen such an HCP within the past year; 9.1\% had never seen an HCP knowledgeable about HHT. Ultimately, $41.3 \%$ of the study population reported having fulfilled all three of the recommended guidelines and were categorized as "high adherers"; the $35.3 \%$ who reported having neglected at least one guideline completely were categorized as "low adherers."

\section{Predictors of HHT screening adherence}

Multivariate regression analysis revealed positive relationships between screening adherence and the variables susceptibility, emotional representations, and treatment control such that as each increased, the probability of being in a higher category of screening adherence increased. Conversely, a negative relationship was observed between screening adherence and perceived barriers to screening such that as perceived barriers increased, the probability of being in a higher screening-adherence category decreased.

As shown in Table 3, of the potential confounders, level of education was the only one found to be a significant predictor of screening adherence. Participants with a high school diploma/general equivalency diploma or a college degree had statistically significantly higher odds of being in a lower adherence category than participants with a postgraduate level of education. The overall model was significant $\left(X^{2}=60.821\right.$; df $=12 ; P=0.000)$ as compared to a model with no predictors. The final model exhibited a moderate fit with a Cox and Snell pseudo- $\mathrm{R}^{2}$ of 0.212 .

Based on the output of the final model, Supplementary Table S1 online displays the predicted probability of being in different levels of adherence based on each of the predictor variables of susceptibility, barriers, treatment control, and emotional representations, while controlling for all other predictor variables (held constant at respective means; thus, the middle column of the table is identical for each variable at its mean), and the confounding variables education, income, and insurance status.

\section{Perceived barriers to HHT screening}

Two hundred fifty-four participants (79.4\%) completed the open-ended questions regarding barriers to pursuing care from an HCP knowledgeable about HHT, and 267 participants (83.4\%) provided perceived benefits (quantitatively summarized in Supplementary Table S2 online).

The majority of participants who cited no barriers/nothing often cited factors that used to be barriers or that could be, and then said how they were able to overcome them or were in a "fortunate" situation. The next most cited barrier to a knowledgeable HCP was the apparent lack of HCPs perceived as being knowledgeable regarding HHT. Participants also frequently mentioned the inconvenience of travel to an HHTknowledgeable HCP, the time required, scheduling difficulties, and the financial burden. One participant explained the difficulties as follows:

So few doctors are knowledgeable about HHT that it is nearly impossible to find one who knows much about the disease. The nearest center of excellence to my home is about 300 miles... It requires a day to travel there, a day or two of appointments, and a day to travel home. I don't know many people, especially people with medical bills, who can afford to take 4 days off simply to go to the doctor about a nosebleed. (21-year-old woman, low adherer)

Two hundred forty-three participants (75.9\%) completed the open-ended questions regarding factors they consider barriers to having their lungs screened for pulmonary AVMs, and 268 participants reported perceived benefits $(83.8 \%)$. The majority (53.9\%) of participants said that there are no factors that keep them from pursuing lung screening. One participant reported:

"Nothing. If I had to walk to an HHT center I would still make the trip because I can't take that risk." (21-year-old woman, high adherer)

Two hundred fifty-six (80.0\%) completed the open-ended questions regarding factors that they consider barriers to undergoing an MRI of the brain to screen for cerebral AVMs, and 265 participants $(82.8 \%)$ cited perceived benefits. Only three factors that were unique or not as commonly cited when considering lung screening were reported as barriers to pursuing an MRI of the brain. The first was the inability for some participants to undergo an MRI, with eight participants noting that they have metal devices or implants contraindicating the procedure. The second was an increased fear of the procedure itself, due primarily to claustrophobia. The third was a sense of denial, as reported by this participant: 
Getting the screening is admitting that I have a life altering problem possibly. (56-year-old man, low adherer)

\section{DISCUSSION}

This study is the first to explore and quantify factors that influence screening behaviors of adults with HHT. Specifically, this study examined the roles of illness representations and HBM constructs in reported adherence to HHT screening. The rates for CAVM screening, PAVM screening, and HHT annual checkup in this study population were $82.0,67.1$, and $56.5 \%$, respectively. Additionally, $41.3 \%$ of participants reported having fulfilled all three of the recommended guidelines (and were subsequently categorized as "high adherers"), whereas 35.3\% reported having neglected at least one guideline completely (and were categorized as "low adherers"). These results illustrate not only that the overarching problem of nonadherence in chronic medical conditions applies to the HHT population but also that the extent of adherence varies across specific recommendations.

In general, participants viewed their HHT as having serious consequences, and the majority agreed that HHT is a serious condition. Participants had a heightened perception of threat and held strong beliefs regarding the chances of serious complications. However, the participants also reported relatively high levels of self-efficacy and response efficacy, demonstrating confidence in their ability to comply with the recommended screening and that the screening will reduce the perceived threat. This study sample was somewhat split in negative feelings triggered by their condition, whereas feelings of understanding (coherence) regarding HHT were quite high overall. These results provide a glimpse into the previously unexplored perceptions of adults with HHT and demonstrate that these individuals are equipped to overcome the barriers given appropriate resources.

Two unique environmental barriers were the apparent perceived lack of HCPs familiar with and/or knowledgeable about HHT and a perceived lack of effort by HCPs to learn about HHT. Inadequate knowledge about genetic conditions among HCPs who do not specialize in genetics has previously been reported. ${ }^{28-31}$ Bernhardt et al..$^{32}$ observed a similar concern among patients with HHT: many felt that primary-care providers might not be adequately prepared to order and accurately interpret genetic testing for HHT. HHT is thought to be considerably underdiagnosed, ${ }^{33,34}$ and it has long been suggested that wider physician awareness of the condition and its pathology could help increase diagnosis and avoid the risks associated with mismanagement. ${ }^{35}$ Another commonly cited environmental barrier was the existence of only 18 multidisciplinary centers in the United States providing comprehensive care for individuals with $\mathrm{HHT},{ }^{36}$ making it difficult to seek care because of geographic and financial limitations.

Similar emotional and cognitive barriers to screening have been identified for other conditions $s^{2,4-7}$ : fear and anxiety that screening may actually reveal something, distrust in HCPs, lack of information regarding where to go for screening, and perceived discomfort, pain, or fear associated with the screening procedure. One troubling finding was that being seemingly asymptomatic acts as a barrier in the decision to pursue HHT screening. This is a particularly challenging barrier because many of the major complications of HHT (e.g., PAVMs and CAVMs) can remain unrecognized until a possibly life-threatening event occurs and the purpose of screening is to intervene, when appropriate, before any hidden complications manifest.

The most commonly reported benefit was that screening will detect complications early. ${ }^{8}$ Additional perceived benefits to HHT screening included early treatment, prevention of complications, monitoring of already existing AVMs, having more knowledge about the status of one's condition, and the belief that screening can save one's life. Participants reported multiple benefits of having an HCP knowledgeable about HHT, including an enhanced patient-physician relationship, communication, and level of trust.

Both HBM and illness-representation constructs have been shown to be strong predictors of preventive health behaviors, $9,13,18-21,37,38$ and the present study has provided support for this in an effort to explain preventive screening behaviors in adults with HHT. Of the 10 independent variables in the multivariate PLUM analyses (six illness-representation constructs and four HBM constructs), four were found to be independent significant predictors of screening adherence: perceived barriers to screening, perceived susceptibility to the threat of health problems associated with HHT, perceived control over one's treatment, and emotional representations triggered by the condition.

The HBM construct of perceived barriers emerged as the most powerful predictor of HHT screening. When all other variables in the model are held constant at their respective means, having a maximum perception of perceived barriers can increase one's odds of being in the low-adherence group 10-fold. Perceived susceptibility was also found to be an independent predictor of screening adherence, with participants who perceived a sense of vulnerability to the potential complications of HHT being more likely to participate in screening. These findings are consistent with the overall evaluation of the HBM.

The illness-representation constructs of treatment control and emotional representations were also found to be positively related, independent predictors of HHT screening adherence. Regarding the former, participants who perceived very little control over their HHT treatment were less likely to adhere to screening recommendations. Regarding the latter, participants reporting more negative emotions triggered by their HHT (e.g., worry and anxiety) were approximately three times more likely to be in the high-adherence category. Although this may seem counterintuitive, greater negative emotions regarding HHT may act as a motivating force, similar to the increasing perceptions of susceptibility and severity in the HBM. Moreover, strong beliefs that HHT is amenable to control via specific treatments may reinforce or enhance one's motivation to adhere to the recommended treatment. Overall, these findings suggest that both cognitive and emotional factors account for HHT screening behavior in this population. 


\section{Limitations}

Despite attempts at recruiting from several different sources, the majority of participants were recruited through the HHT Foundation International, a nonprofit organization whose purpose is to support and educate patients, families, and medical professionals. Because the majority of participants were most likely members of the foundation, it is quite possible that the sample represents a group of individuals relatively knowledgeable about HHT. In addition, the study population was largely female and Caucasian, which does not proportionately represent the population of individuals with HHT. Moreover, participants were asked to self-report their screening behaviors, introducing the potential for recall bias; it is possible that selfreport measures overestimate adherence, especially when compared with more direct measures. ${ }^{39}$ Finally, many of the HBM constructs and IPQ-R variables are significantly correlated with each other (see Supplementary Table S3 online). We acknowledge that the possibility of multicollinearity may increase the standard errors of the coefficients, potentially obscuring an effect.

\section{Clinical implications}

The results of this study reveal several important concepts for clinical practice in the HHT population. First, it is important for clinicians caring for patients with HHT to know that screening rates are not ideal and that confirming the diagnosis and making screening recommendations do not necessarily result in adherence. The screening behaviors in this population are complex, with many factors contributing to the decision to pursue screening. Second, approximately one-third of participants reported being unable to find an HCP familiar with HHT, which is especially disheartening given that the majority of participants were recruited through one of the primary patient resources available for individuals with HHT. Approximately $9 \%$ of participants who provided a response regarding perceived benefits of seeing a provider knowledgeable about HHT reported assuming the role of "teacher" within their patientphysician relationship, including initiating discussions regarding their HHT treatments and necessary screening.

There are several possible approaches to improving screening adherence by attempting to reduce barriers and taking into account perceived susceptibility. Our findings demonstrate the need for further physician education, which can be provided through organizations specializing in HHT and/or genetics, such as the HHT Foundation International and the American College of Medical Genetics and Genomics. As suggested by Latino et al., ${ }^{33}$ ENT physicians may prove to be an excellent physician group to initially target with educational programs to improve diagnostic rates of HHT.

In addition, it may be beneficial to assess a patient's understanding of the potential complications of AVMs. If the patient is not fully aware of the possible consequences, the information should be presented in balance with encouragement and an optimistic perspective of his or her ability to influence such outcomes of HHT by adhering to the recommended screening guidelines. Recent research shows that the life expectancy of HHT patients may differ based on the molecular basis of the disease. ${ }^{40}$ It would be prudent for future HHT research and medical providers advising patients about HHT screening to consider the underlying genetic defect.

\section{SUPPLEMENTARY MATERIAL}

Supplementary material is linked to the online version of the paper at http://www.nature.com/gim

\section{ACKNOWLEDGMENTS}

The authors thank all the adults with HHT who completed our survey because their efforts were essential to the study. They also thank Alexis Heidlebaugh, Kendall Umstead, and Barbara Biesecker for formatting and editing the manuscript for publication. The National Human Genome Research Institute Intramural Research Program, National Institutes of Health, funded this study.

\section{DISCLOSURE}

M.B. holds stock in Informed Medical Decisions, Inc. The other authors declare no conflict of interest.

\section{REFERENCES}

1. Bayrak-Toydemir P, Mao R, Lewin S, McDonald J. Hereditary hemorrhagic telangiectasia: an overview of diagnosis and management in the molecular era for clinicians. Genet Med 2004;6:175-191.

2. Faughnan ME, Palda VA, Garcia-Tsao G, et al.; HHT Foundation InternationalGuidelines Working Group. International guidelines for the diagnosis and management of hereditary haemorrhagic telangiectasia. J Med Genet 2011;48:73-87.

3. DiMatteo MR. Variations in patients' adherence to medical recommendations: a quantitative review of 50 years of research. Med Care 2004;42:200-209.

4. Consedine NS, Magai C, Krivoshekova YS, Ryzewicz L, Neugut Al. Fear, anxiety, worry, and breast cancer screening behavior: a critical review. Cancer Epidemiol Biomarkers Prev 2004;13:501-510.

5. Bleiker EM, Menko FH, Taal BG, et al. Screening behavior of individuals at high risk for colorectal cancer. Gastroenterology 2005;128:280-287.

6. Farraye FA, Wong M, Hurwitz S, et al. Barriers to endoscopic colorectal cancer screening: are women different from men? Am J Gastroenterol 2004;99: 341-349.

7. Thompson B, Montaño DE, Mahloch J, Mullen M, Taylor V. Attitudes and beliefs toward mammography among women using an urban public hospital. J Health Care Poor Underserved 1997;8:186-201.

8. Byrd TL, Chavez R, Wilson KM. Barriers and facilitators of cervical cancer screening among Hispanic women. Ethn Dis 2007;17:129-134.

9. Harewood GC, Wiersema MJ, Melton LJ 3rd. A prospective, controlled assessment of factors influencing acceptance of screening colonoscopy. Am J Gastroenterol 2002;97:3186-3194.

10. Rustgi AK. Breaking the barriers to colorectal cancer screening. Gastroenterology 2004;126:1232-1233.

11. Taheri-Kharameh Z, Noorizadeh F, Sangy S, Zamanian H, Shouri-Bidgoli AR, Oveisi $\mathrm{H}$. Factors associated with adherence to colorectal cancer screening among moderate risk individuals in Iran. Asian Pac J Cancer Prev 2015;16: 8371-8375.

12. Khdour MR, Hawwa AF, Kidney JC, Smyth BM, McElnay JC. Potential risk factors for medication non-adherence in patients with chronic obstructive pulmonary disease (COPD). Eur J Clin Pharmacol 2012;68:1365-1373.

13. du Treil S, Rice J, Leissinger CA. Quantifying adherence to treatment and its relationship to quality of life in a well-characterized haemophilia population. Haemophilia 2007;13:493-501.

14. Bernard RS, Cohen LL. Increasing adherence to cystic fibrosis treatment: a systematic review of behavioral techniques. Pediatr Pulmonol 2004;37:8-16.

15. Janz NK, Champion VL, Strecher VJ. The Health Belief Model. In: Glanz K, Rimer BK, Lewis FM (eds). Health Behavior and Health Education: Theory, Research, and Practice, 3rd edn. Jossey-Bass: San Francisco, CA, 2002:45-66. 
16. Alatawi YM, Kavookjian J, Ekong G, Alrayees MM. The association between health beliefs and medication adherence among patients with type 2 diabetes. Res Social Adm Pharm 2015; e-pub ahead of print 22 November 2015.

17. Khouzam A, Kwan A, Baxter S, Bernstein JA. Factors associated with uptake of genetics services for hypertrophic cardiomyopathy. J Genet Couns 2015;24:797-809.

18. Palmer RC, Emmons KM, Fletcher $\mathrm{RH}$, et al. Familial risk and colorectal cancer screening health beliefs and attitudes in an insured population. Prev Med 2007:45:336-341.

19. Verkerk MM, Shovlin CL, Lund VJ. Silent threat? A retrospective study of screening practices for pulmonary arteriovenous malformations in patients with hereditary haemorrhagic telangiectasia. Rhinology 2012;50:277-283.

20. Rosenstock IM, Strecher VJ, Becker MH. Social learning theory and the Health Belief Model. Health Educ Q 1988;15:175-183.

21. Moss-Morris R, Petrie KJ, Horne R, Cameron LD, Buick D. The revised illness perception questionnaire (IPQ-R). Psychol Health 2002;17:1-16.

22. Green PM, Kelly BA. Colorectal cancer knowledge, perceptions, and behaviors in African Americans. Cancer Nurs 2004;27:206-15; quiz 216.

23. Champion $V L$, Scott CR. Reliability and validity of breast cancer screening belief scales in African American women. Nurs Res 1997;46:331-337.

24. Champion V, Skinner CS, Menon U. Development of a self-efficacy scale for mammography. Res Nurs Health 2005;28:329-336.

25. Skinner CS, Arfken CL, Sykes RK. Knowledge, perceptions, and mammography stage of adoption among older urban women. Am J Prev Med 1998;14:54-63.

26. Plotnikoff RC. Protection motivation theory and exercise behavior change for the prevention of coronary heart disease in a high-risk, Australian representative community sample of adults. Psychol Health Med 2002;71:87-98.

27. O'Connell A. Logistic Regression Models for Ordinal Response Variables. Sage Publications: Thousand Oaks, CA, 2006.

28. Scheuner MT, Sieverding P, Shekelle PG. Delivery of genomic medicine for common chronic adult diseases: a systematic review. JAMA 2008;299:1320-1334.
29. Suther S, Goodson P. Barriers to the provision of genetic services by primary care physicians: a systematic review of the literature. Genet Med 2003;5: 70-76.

30. Harvey EK, Fogel CE, Peyrot M, Christensen KD, Terry SF, Mclnerney JD. Providers' knowledge of genetics: a survey of 5915 individuals and families with genetic conditions. Genet Med 2007;9:259-267.

31. Acton RT, Barton JC, Casebeer L, Talley L. Survey of physician knowledge about hemochromatosis. Genet Med 2002:4:136-141.

32. Bernhardt BA, Zayac C, Pyeritz RE. Why is genetic screening for autosomal dominant disorders underused in families? The case of hereditary hemorrhagic telangiectasia. Genet Med 2011;13:812-820.

33. Latino GA, Brown D, Glazier RH, Weyman JT, Faughnan ME. Targeting underdiagnosis in hereditary hemorrhagic telangiectasia: a model approach for rare diseases? Orphanet J Rare Dis 2014;9:115.

34. Grosse SD, Boulet SL, Grant AM, Hulihan MM, Faughnan ME. The use of US health insurance data for surveillance of rare disorders: hereditary hemorrhagic telangiectasia. Genet Med 2014;16:33-39.

35. Guttmacher AE, Marchuk DA, White RI Jr. Hereditary hemorrhagic telangiectasia. N Engl J Med 1995;333:918-924.

36. HHT Foundation International. North American HHT Centers of Excellence. 2015. https://directory.curehht.org/hht-centers. Accessed 28 January 2016.

37. Jessop DC, Rutter DR. Adherence to asthma medication: the role of illness representations. Psychol Health 2003;18:595-612.

38. Griva K, Myers LB, Newman S. Illness perceptions and self efficacy beliefs in adolescents and young adults with insulin dependent diabetes mellitus. Psychol Health 2000;15:733-50.

39. Dunbar-Jacob J, Schlenk E. Patient adherence to treatment regimen. In: Baum A, Revenson TA, Singer JE (eds). Handbook of Health Psychology. Lawrence Erlbaum Associates: Mahwah, NJ, 2001:571-580.

40. de Gussem EM, Edwards CP, Hosman AE, et al. Life expextancy of parents with hereditary haemorrhagic telangiectasia. Orphanet J Rare Dis 2016;11:46. 